\title{
A Rare Case of Extra-Adrenal Pheochromocytoma
}

\author{
Dr.Ramesh Kumar Korumilli ${ }^{1}$, Dr.Victor Vinod Babu ${ }^{2}$, Dr.G.Gautam Reddy ${ }^{3}$ \\ ${ }^{I}$ (professor, department of general surgery, svs medical college, Dr.Ntr university of health sciences, India) \\ 2(department of general surgery, Dr.Ntr university of health sciences, India) \\ ${ }^{3}$ (assistant professor, department of general surgery,svs medical college, Dr.Ntr university of health sciences,
} India)

\begin{abstract}
Extra-adrenal pheochromocytoma develops in paraganglion chromaffin cells of the sympathetic nervous system. It probably represents at least $15 \%$ of adult and $30 \%$ of childhood pheochromocytomas. In this we report, a young female of 22 years who presented with headache of 6 yrs and uncontrolled hypertension of 1 yr. Before coming to surgical OPD she was being managed by physician, cardiologist, ophthalmologist, neurophysician. As she had mild abdominal discomfort careful examination revealead a mass which on detailed investigation confirmed the diagnosis of extra-adrenal pheochromocytoma. Resection resulted in complete relief of symptoms and control of hypertension.
\end{abstract}

Keywords: extra-adrenal, headache, hypertension, pheochromocytoma

\section{Introduction}

Extra-adrenal pheochromocytomas (EAPs) may arise in any portion of the paraganglion system, though they most commonly occur below the diaphragm, frequently in the organ of Zuckerkandl. They may be malignant in upto $40 \%$ of the cases, though conflicting data add to the uncertainty of this point.

\section{П. Case Report}

A female patient of $22 \mathrm{yrs}$ of age presented to the surgical out patient department with mild abdominal discomfort, no vomiting, bowel habits normal. History revealed she was being treated by ophthalmologist for vision problems and headache for the past 6 yrs, she was found to be hypertensive and under treatment by physician and cardiologist for the past $1 \mathrm{yr}$. She was getting consultation of neurophysician for headache and an episode of seizures. Pulse rate was 120/min, blood pressure of 160/120 mm of Hg. Examination of abdomen revealed a small mass in the right lumbar area. USG and CECT abdomen revealed a $4.5 \times 3.7 \mathrm{~cm}$ heterogenous lesion, abutting the head of pancreas, in front of aorta and inferior vena cava (Fig.1).

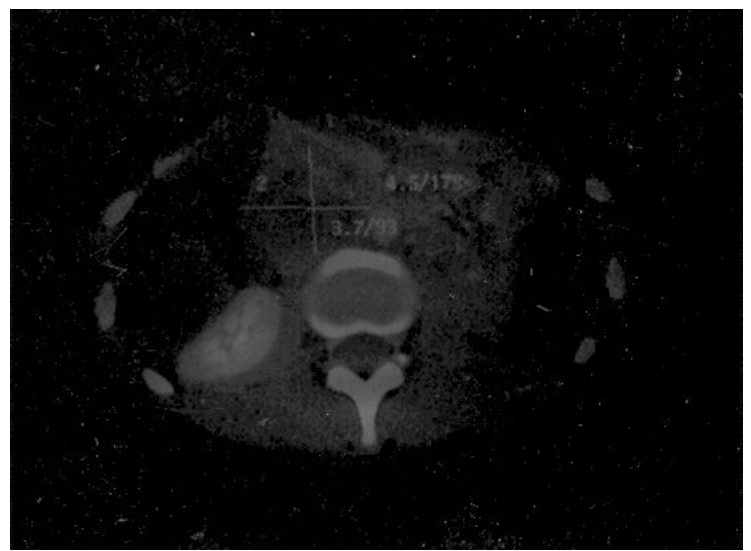

Fig.1 CT scan abdomen showing tumor mass

MIBG scan with I 131 revealed paraganglioma or pheochromocytoma. $24 \mathrm{hrs}$ urine examination showed elevated metanephrines, VMA and proteins. Retinal examination revealed grade 1 hypertensive retinopathy. Cardiovascular examination was normal. Planned for surgery and risks were explained to the patient. Preoperative preparation of the patient and control of hypertension was done in consultation with anaesthetist with nifedipine, aten and then with doxacard. Patient was taken up for surgery. Through midline incision abdomen was opened, kocherisation of the duodenum done. A $4 \times 3 \mathrm{~cm}$ tumor was found over IVC and aorta at L2-L4 level. Tumor was excised with slow and steady dissection after ligating all feeding vessels(Fig.2). 


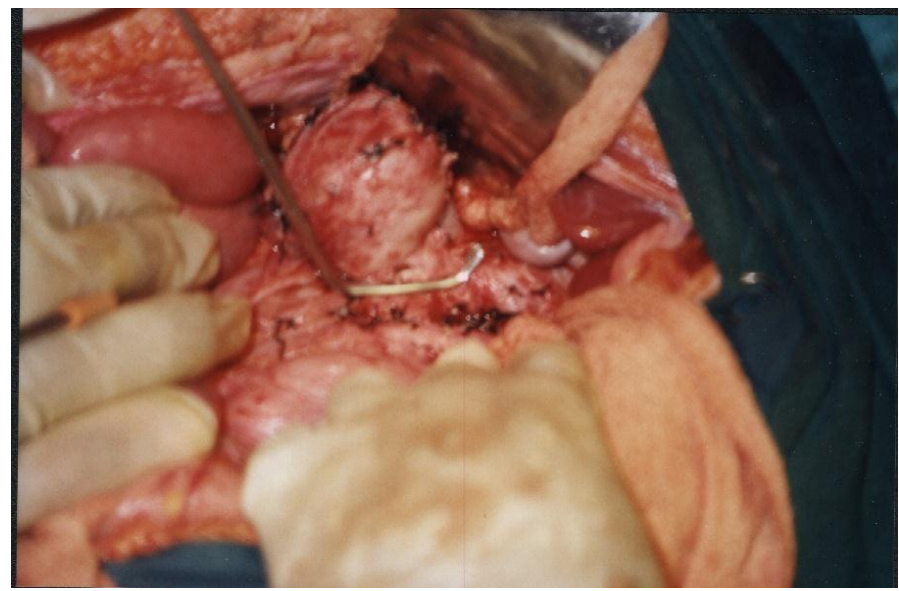

Fig.2 intra-operative photograph showing tumor mass

Blood pressure was maintained within normal limits throughout the procedure. Post-operative period was uneventful with blood pressure maintained within normal limits. Aten $25 \mathrm{mg}$ continued for a week.Histopathology report confirmed the diagnosis of pheochromocytoma(Fig.3)

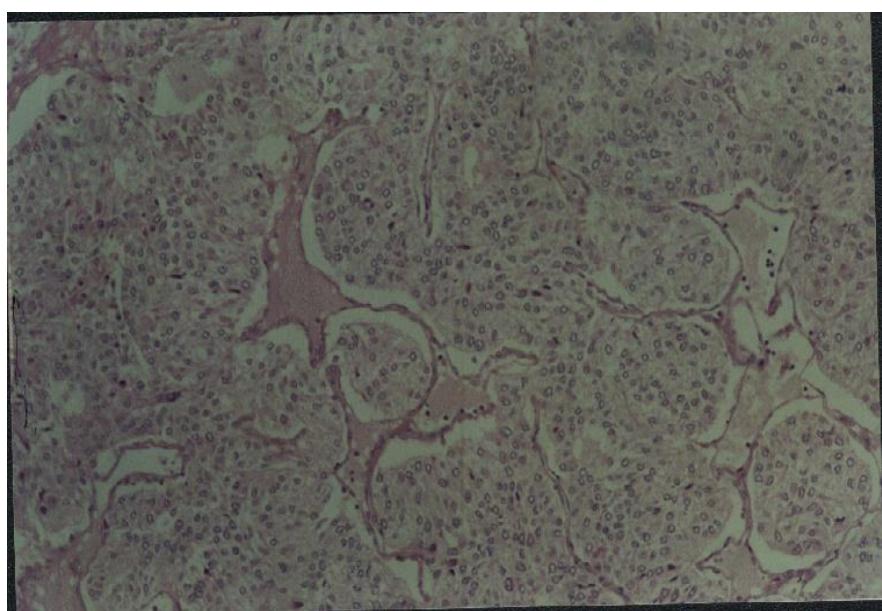

Fig.3 Histopathological picture of pheochromocytoma

At the time of discharge after 1 week her blood pressure was 120/80 $\mathrm{mm}$ of $\mathrm{Hg}$ and also relieved of headache. During monthly follow-up for four months her blood pressure was within normal limits without any antihypertensive drugs. After 1 yr. she came for review, was asymptomatic and maintaining normal blood pressure without any drugs.

\section{Ш. Discussion}

Pheochromocytoma is rare, accounting for less than $0.1 \%$ of hypertensive population. Extra-adrenal pheochromocytomas are rarer still (10\% of all pheochromocytomas) and are usually seen in the second and third decades $^{[2]}$. There is no sex predominance ${ }^{[1]}$. Extra-adrenal pheochromocytomas are often multicentric and more likely to be malignant than those of adrenal origin ${ }^{[1]}$.

Although more properly known as paragangliomas, today these tumors are frequently called extraadrenal pheochromocytomas (EAPs). The traditional teaching of the " $10 \%$ rule," which noted that $10 \%$ of all pheochromocytomas are at extra-adrenal sites, may actually be an underestimation. A review of the literature suggests that EAPs actually constitute $15 \%$ of adult and $30 \%$ of pediatric pheochromocytomas ${ }^{[1]}$. These tumors can arise wherever the cells of the paraganglionic system are located. Paraganglia are chromaffin tissue complexes of the neuroendocrine system distributed along the paravertebral and para-aortic axes, extending from the cervical region down to the base of the pelvis. The collection of paraganglia located anterolaterally to the distal abdominal aorta between the origin of the inferior mesenteric artery and the aortic bifurcation is called the organ of Zuckerkandl. Eighty-five percent of EAPs are located in the retroperitoneum, usually arising from the organ of Zuckerkandl. Less common sites reported include the bladder, thorax, neck, and pelvis ${ }^{[3]}$. 
Patients with EAPs may present with headache, palpitations, sweating, or hypertension. A small percent of patients may also be asymptomatic at presentation due to non-functional tumors. The diagnosis is confirmed by demonstrating elevated blood and urine levels of catecholamines and their metabolites ${ }^{[4]}$. Imaging studies to evaluate for EAPs include CT, MRI, and 131I-labelled metaiodobenzylguanidine scintigraphy. Preoperative pharmacologic preparation, attentive intraoperative monitoring, and aggressive surgical therapy have important roles in achieving successful outcomes. EAPs recur and metastasize more often than their adrenal counterparts, making lifelong follow-up essential.

\section{IṼ. Conclusion}

This patient suffered without proper diagnosis and treatment for a long period inspite of visiting various specialists. Because of the rarity, thorough physical examination, investigations are essential for diagnosis. Good preoperative preparation, teamwork along with good anaesthetic management both preoperative and intra-operative and effective post-operative care are essential for successful outcome. A lifelong follow-up is indicated, as the extra-adrenal pheochromocytomas are more likely to recur and to metastasize.

\section{References}

[1]. Whalen RK, Althausen AF, Daniels GH. Extra-adrenal pheochromocytoma. J Urol 1992: 147: 1-10.

[2]. Goldfarb DA, Novick AC. Bravo L et al. Experience with extra adrenal pheochromocytorna. J Urol 1989; 142: 931-936.

[3]. Salanatri J, Smith P, Sclicht S: Multifocal malignant extra-adrenal paragangliomas of the organ of Zuckerlandl and urinary bladder. Austral Radiol 2001, 45: 229-232.

[4]. Lenders J, Pacak K, Walther W, et al: Biochemical diagnosis of pheochromocytomas: which test is best? JAMA 2002, 287:14271434. 